\title{
Effects of atmospheric electric fields on detection of ultrahigh-energy cosmic rays
}

\author{
Alexander Kusenko and Dmitry Semikoz \\ Department of Physics and Astronomy, UCLA, Los Angeles, CA 90095-1547, USA
}

\begin{abstract}
We show that atmospheric electric fields may affect the cosmic ray observations in several ways and may lead to an overestimation of the cosmic ray energies. The electric field in thunderclouds can be as high as a few $\mathrm{kV} / \mathrm{cm}$. This field can accelerate the shower electrons and can feed some additional energy into the shower. Therefore, ground array observations in certain weather conditions may overestimate the energy of ultrahigh-energy cosmic rays if they don't take this effect into account. In addition, the electric field can bend the muon trajectories and affect the direction and energy reconstruction of inclined showers. Finally, there is a possibility of an avalanche multiplication of the shower electrons due to a runaway breakdown, which may lead to a significant miscalculation of the cosmic ray energy.
\end{abstract}

PACS numbers: 96.40.-z, 95.55.Vj, 95.85.Ry, 98.70.Sa

UCLA/04/TEP/44

\section{INTRODUCTION}

Ultrahigh-energy cosmic rays (UHECR) produce extensive air showers in the atmosphere, which are observed by two different techniques: (1) optical detection of fluorescent light, and (2) surface array detection of charged particles in the shower. The first technique, employed, for example, by HiRes detector [1], can be used exclusively in good weather, while the second technique, used, for example, by the AGASA experiment [2], is usually believed to afford accurate observations regardless of the atmospheric conditions. The new Pierre Auger experiment is using a combination of the two techniques [3].

On a clear day the atmosphere is permeated by the electric fields of order a few volts per $\mathrm{cm}$ [4]. However, in thunderclouds the electric field reaches much higher magnitudes, up to a few $\mathrm{kV} / \mathrm{cm}[5]$. Balloon measurements [6] show the electric fields $\mathcal{E}>1 \mathrm{kV} / \mathrm{cm}$, at altitudes $0-12$ $\mathrm{km}$, which may switch polarity at several altitudes. A shower developing in the atmosphere and going through a thundercloud may, therefore, pass between layers with either polarity at some angle. The electric field along the path of a random shower may have a magnitude of a few $\mathrm{kV} / \mathrm{cm}$ and an arbitrary direction, or alternating direction. As we discuss below, the electron energy gain in the electric field of a few $\mathrm{kV} / \mathrm{cm}$ can be comparable to the energy losses due to ionization. It, therefore, important to examine what effect this may have on detection of UHECR.

Several experimental studies confirm the influence of electric fields on secondary particles in a shower. First, observations of low-energy cosmic ray electrons show short-term variations during thunderstorms 7]. A similar effect has been observed for muons with a higher energy, $E>100 \mathrm{MeV}$ [7, 8], but it is not nearly as strong as the effect on the low-energy electrons. Second, it has been established that giant electron-gamma bursts are triggered in thunderclouds by the passage of an extensive air shower (EAS) from a $10^{16}$ cosmic ray [9]. EAS were measured at the Tien Shan Mountain in coincidence with detection of a radio signal from the giant electron-gamma bursts. Theory of these bursts, based on the phenomenon of runaway breakdown, has been developed in recent years [10]. It has was also suggested that acceleration of secondary electrons from EAS in thunderclouds can be used for radio detection of cosmic rays with energies $E>10^{17-19} \mathrm{eV}[11$.

We will show that the atmospheric electricity associated with thunderclouds may affect the ground array observations of $E>10^{19-20} \mathrm{eV}$ cosmic rays in several ways. First, acceleration of the shower electrons can alter the low-energy spectrum and the number of particles in EAS at ground level. This could lead to errors in energy determination by a ground array. Second, the muon trajectories may be deflected by the atmospheric electric fields, which could lead to errors in reconstruction of energy and direction of inclined showers. Finally, a runaway breakdown and discharge triggered by an UHECR EAS may produce a dramatic increase in the number of electrons detected by the surface array.

\section{ENERGY OF THE ELECTROMAGNETIC COMPONENT}

An electron or positron with momentum $\vec{p}=\vec{e}_{x} p_{x}+$ $\vec{e}_{y} p_{y}$ moving in the atmospheric field $\mathcal{E}(x, y)$ is described by the following system of equations:

$$
\left\{\begin{array}{l}
\dot{p_{x}}=-\kappa(y) \frac{p_{x}}{p} \\
\dot{p_{y}}=e \mathcal{E}(x, y)-\kappa(y) \frac{p_{y}}{p}
\end{array}\right.
$$

where $\kappa$ accounts for the ionization losses. Here we have assumed $\kappa$ to be independent of energy (a good approximation for the shower electrons). Of course, $\kappa$ depends on the air density, and, hence, on the height $y$ in the atmosphere. The electric field is also a function of coordinates.

For a vertical shower in a vertical electric field $\mathcal{E}=$ $\mathcal{E}(y)$, the horizontal component of the momentum vanishes, $p_{x}=0$, and the effect of the electric field amounts to reducing the energy losses of the shower in the atmo- 

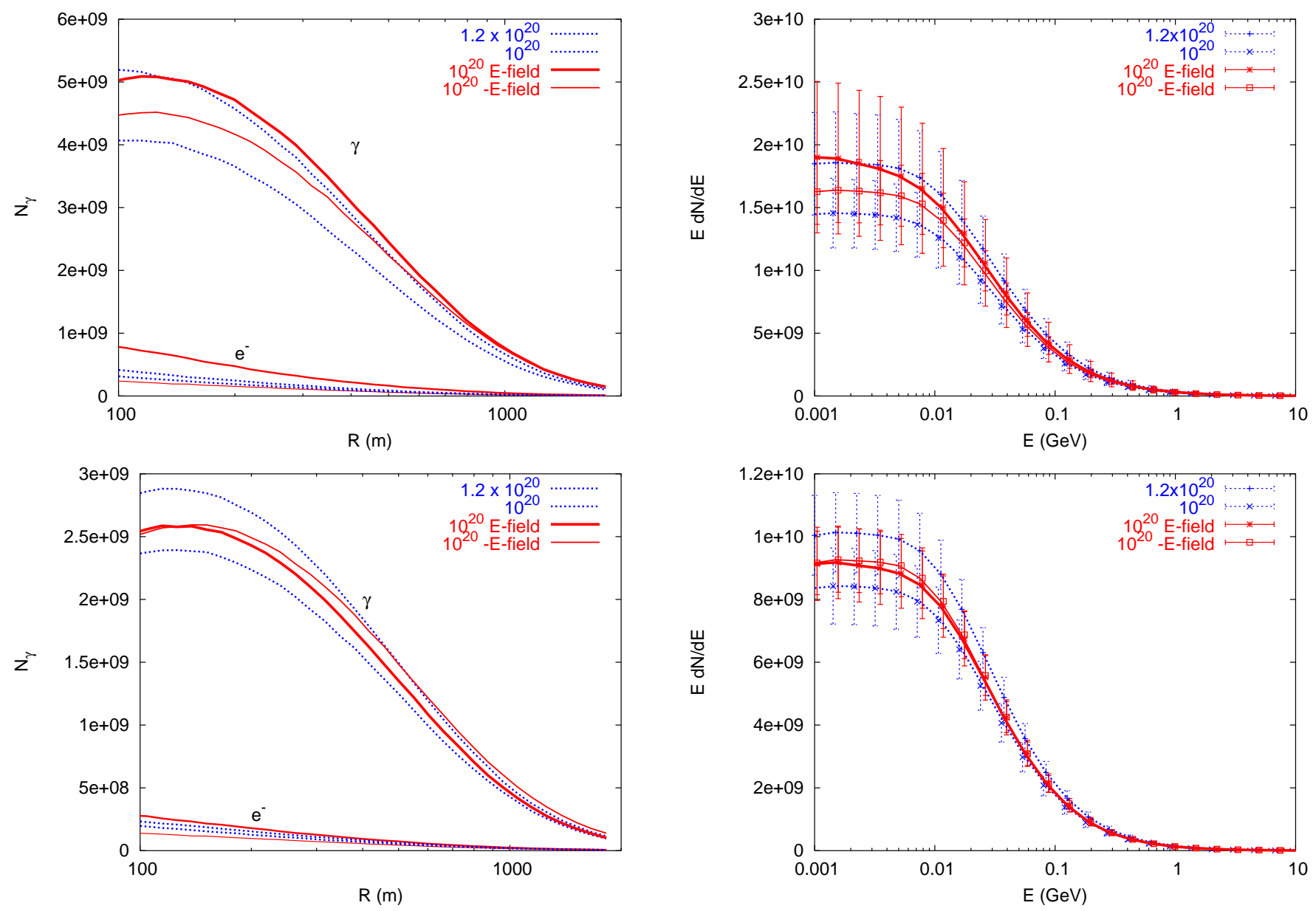

FIG. 1: Effect of a uniform atmospheric electric field on the shower profile for a proton primary (upper plot) and an iron primary (lower plot). The dotted lines show AIRES simulations of the $10^{20} \mathrm{eV}$ (low line) and $1.2 \times 10^{20} \mathrm{eV}$ (upper line) showers, respectively, in the absence of atmospheric electric field. The thick and thin solid lines correspond to a $10^{20} \mathrm{eV}$ shower developing in the presence of a uniform electric field $\pm 1 \mathrm{kV} / \mathrm{cm}$. The electric field makes the $10^{20} \mathrm{eV}$ shower look more like a $(1.1-1.2) \times 10^{20} \mathrm{eV}$ shower.

sphere. The equation of motion is

$$
\dot{p_{y}} \approx \frac{d}{d y} p_{y}=e \mathcal{E}(y)-\kappa(y)
$$

where we have taken, as usual $c=1$. The solution of this equation,

$$
p_{y}(y)=p_{y, 0}-\int_{y_{0}}^{y}(e \mathcal{E}(\xi)-\kappa(\xi)) d \xi,
$$

depends on the initial momentum of an electron and on the form of $(e \mathcal{E}(y)-\kappa(y))$, where $\kappa(y)$ is a function of density only. From the form of this solution it is clear that, for a vertical shower, the electric field can reduce the energy losses and to make the atmosphere effectively

FIG. 2: Effect of atmospheric electric field on the photon and electron spectra. The the dotted lines represent the spectra of $10^{20} \mathrm{eV}$ and $1.2 \times 10^{20} \mathrm{eV}$ showers, respectively, in the absence of atmospheric electric field. The thick and thin solid lines correspond to a $10^{20} \mathrm{eV}$ shower developing in the presence of a uniform electric field $\pm 1 \mathrm{kV} / \mathrm{cm}$. In the presence of electric field, the apparent energy of the shower is higher than it's actual energy.

thinner. Of course, if the electric field is larger than $\kappa / e$, the electron gains energy faster than it loses energy. This possibility will be discussed below.

The energy losses of the shower electrons due to ionization are

$$
\kappa=\frac{d E}{d y} \approx\left(\frac{\rho}{0.0013 \mathrm{~g} \mathrm{~cm}^{-3}}\right)\left[\ln \frac{E}{m_{e}}+6.3\right] \frac{\mathrm{keV}}{\mathrm{cm}} .
$$

This should be compared with the atmospheric electric field, which reaches the values

$$
\mathcal{E} \gtrsim 1 \frac{\mathrm{kV}}{\mathrm{cm}}
$$

in thunderclouds [5]. Obviously, the effect of the electric field is non-negligible.

The experimental determination of UHECR energy is based on the reconstruction of the total energy of photons and electrons at the shower maximum based on the 
observed total energy at ground level. The extrapolation to shower maximum involves, effectively, solving the evolution of shower energy due to the energy losses as well as the change in the spectrum and the number of particles due to a number of elementary processes. Most of the particles reaching the ground are photons; in particular, there are more photons than electrons at ground level by an order of magnitude. These photons originate from (i) bremsstrahlung of electrons, (ii) annihilations of positrons. If the electrons are accelerated by the field, the positrons are slowed down, and vise versa. The electric field along the path of a shower can have either polarity. The electric field may also have different directions at different heights [5]. On average, there is more positive charge in the higher layers of the atmosphere, and the ionosphere is almost always positively charged. However, the thunderclouds is where the field reaches a high magnitude. The electric field in a cloud is not necessarily uniform. It can have arbitrary direction along the shower path.

If the electrons are accelerated, the atmosphere is effectively thinner for them. Therefore, these electrons can produce the bremsstrahlung photons over a longer period of time, and the resulting photons have, on average, a higher energy. If positrons are accelerated, they have a higher average energy when they annihilate, and, in addition, they annihilate closer to the ground, on average. These are independent and, perhaps, competing effects, so a priori it is difficult to know which of them dominates. In addition, one has to keep in mind that the ground detectors are sensitive to particles about $0.6-1.2 \mathrm{~km}$ away from the shower axis. It is difficult to evaluate the overall effect of the electric fields analytically. We have employed a numerical calculation using AIRES [12] software package to simulate the effects of the atmospheric electric field. To this end we changed the electron and positron energy loss rate in accordance with eq. (3), assuming a constant electric field along the direction of the shower. It turns out that both the acceleration of electrons and positrons give similar effect.

We illustrate the effect in Figures 1 1 and In Fig. 1 we show the number of photons and electrons reaching the ground as a function of distance from the shower core. The upper plot corresponds to a proton primary, while lower plot is for iron. Dotted (blue) lines present shower profiles with a primary energy $10^{20} \mathrm{eV}$ (lower line) and $1.2 \times 10^{20} \mathrm{eV}$ (upper line). Thick (thin) solid line corresponds to the case of a $10^{20} \mathrm{eV}$ shower affected by a $1 \mathrm{keV} / \mathrm{cm}$ electric field in the direction for which the electrons (positrons) are accelerated. One can see that, in the presence of the electric field, the number of photons increases, and the $10^{20} \mathrm{eV}$ shower begins to resemble a $1.2 \times 10^{20} \mathrm{eV}$ shower. Although it makes a difference whether electrons or positrons are accelerated (decelerated), the overall effect increases the apparent shower energy in both cases. One can also see from Fig. 1 that number of electrons and positrons reaching the ground changes with the direction of the field, but that their number in any case is negligible in comparison with the number of photons.

In Fig. 2 we show the energy distribution of photons and electrons in the shower at ground level. The curve markings are similar to those in Fig. 1 In the presence of an electric field, the apparent shower energy may seem higher than the actual energy by as much as $20 \%$.

We emphasize that our simulations were very crude: we assumed a uniform electric field along the shower path. In reality, the electric field configuration can be much more complicated, and this can either increase or decrease the significance of the effect. Also, in these numerical simulations we neglected the effect of an electric field on the muon trajectories. More detailed simulations are required for more reliable results. It would be desirable to measure the electric fields above the experimental site and use these measurements in the data analysis. Measuring the electric field at ground level is relatively straightforward.

\section{MUON ARRIVAL DIRECTIONS}

The Pierre Auger detector is sensitive to both electrons and muons in the shower. For a highly inclined shower, the electromagnetic component dies out before the shower reaches the ground, and, therefore, the data analysis is based on muons alone. The arrival times of muons are used to reconstruct the angle of the shower. Here the effects of the atmospheric electric field must also be taken into account because the electric field bends the muon trajectories.

Let us approximate $\kappa$ by a constant (constant density atmosphere), and let us consider a uniform electric field. For a small field, the angle is approximately constant and is close to the initial value $\theta_{0}$,

$$
\tan \theta_{0}=\frac{p_{x, 0}}{p_{y, 0}} .
$$

As long as this is the case, the equations (1) have an approximate solution

$$
\left\{\begin{array}{l}
p_{x}=p_{x, 0}-\kappa \sin \theta_{0} t \\
p_{y}=p_{y, 0}+e \mathcal{E} t-\kappa \cos \theta_{0} t
\end{array}\right.
$$

The components of the particle's velocity are

$$
\begin{aligned}
& \dot{x}=\frac{p_{x}}{\sqrt{m^{2}+p^{2}}} \\
& \dot{y}=\frac{p_{y}}{\sqrt{m^{2}+p^{2}}},
\end{aligned}
$$

where, as before, $p=\sqrt{p_{x}^{2}+p_{y}^{2}}$. For the zenith angle one can write

$$
\operatorname{cotan} \theta=\frac{d y}{d x}=\frac{\dot{y}}{\dot{x}}=\frac{p_{y}}{p_{x}}
$$


Using the approximate solution (7), we get

$$
\begin{aligned}
\dot{\theta} & =\frac{d}{d t} \arctan \left(\frac{p_{x}}{p_{y}}\right) \\
& =\frac{p_{x, 0}\left(e \mathcal{E}-\cos \theta_{0} \kappa\right)+p_{y, 0} \sin \theta_{0}}{p_{0}^{2}+2 A t+B t^{2}},
\end{aligned}
$$

where $A=\left(e \mathcal{E}-\cos \theta_{0} \kappa\right) p_{y, 0}-\kappa \sin \theta_{0} p_{x, 0}$ and $B=$ $e^{2} E^{2}-2 e E \kappa \cos \theta_{0}+\kappa^{2}$. Therefore, the change in the angle is of the order of

$$
\Delta \theta \sim \frac{e \mathcal{E} t}{p} \sim 10^{-1}\left(\frac{3 \mathrm{GeV}}{p}\right)\left(\frac{e E}{1 \frac{\mathrm{kV}}{\mathrm{cm}}}\right)\left(\frac{c t}{3 \mathrm{~km}}\right)
$$

for a uniform electric field $E$. This change is nonnegligible. It introduces an error in the angle reconstruction of the arrival direction. In addition, if one uses muons for energy estimation, this effect can introduce a systematic error because one could miscalculate the length of the shower path in the atmosphere.

\section{RUNAWAY BREAKDOWN}

When the electric field in a thundercloud reaches some critical value of the order a few $\mathrm{keV}$, a runaway breakdown may generate a large number of avalanche electrons with energies in the $0.1-10 \mathrm{MeV}$ range [10]. The runaway electron avalanches are triggered by the seed electrons in the extensive air showers initiated by the cosmic rays of energies $10^{16} \mathrm{eV}$ and higher 10 . There is a mounting evidence that this form of discharge is the reason why the electric field in thunderclouds is limited to a few $\mathrm{keV}$. In the absence of cosmic rays the field could reach values that are an order of magnitude greater than those observed in clouds.

If an electron avalanche accompanies an air shower observed by the ground array, the number of electrons and photons registered by the detector could increase significantly, and the cosmic ray energy can be greatly overestimated.
The runaway breakdown requires the electric field to reach a critical value [10], which is

$$
\mathcal{E}_{c} \approx \frac{44 \pi e^{3} Z n}{m_{e}} \approx 2.2 \frac{\mathrm{kV}}{\mathrm{cm}}
$$

in the lower atmosphere. Here $n$ and $Z$ are the molecular density and the electron number, respectively. Since $10^{16} \mathrm{eV}$ showers are apparently sufficient to discharge the cloud, the field is unlikely to be critical at the time and place of a (much less frequent) $10^{18}-10^{20} \mathrm{eV}$ shower. However, this probability is non-negligible and it is possible that some of the high-energy showers can appear much more energetic to the ground array. This effect should be taken into account by cosmic ray experiments.

\section{CONCLUSION}

We have shown that atmospheric electric fields may affect the energy measurements by the ground arrays in certain weather conditions. Our simplified calculations show that, in the presence of thunderclouds, one may overestimate the shower energy by as much as $20 \%$. In addition, the deflection of muons may affect the directional reconstruction of inclined showers, which relies on muon arrival times. Finally, if an UHECR shower is accompanied by a runaway breakdown, the number of shower electrons can increase dramatically, which may lead to a significant miscalculation of the cosmic ray energy.

\section{Acknowledgments}

We thank K. Arisaka, J. Lee, T. Ohnuki, A. Tripathi, and the rest of the Pierre Auger group at UCLA for numerous discussions and comments. We also thank V. Rubakov for helpful comments. This work was supported in part by the DOE grant DE-FG03-91ER40662 and the NASA ATP grant NAG 5-13399.
[1] R. U. Abbasi et al. [High Resolution Fly's Eye Collaboration], Phys. Rev. Lett. 92, 151101 (2004) arXiv:astro-ph/0208243.

[2] S. Yoshida et al., Astropart. Phys. 3, 105 (1995).

[3] L. A. Anchordoqui [The AUGER Collaboration], arXiv:astro-ph/0409470

[4] R.G. Fleagle and J.A.Businger, An introduction to atmospheric physics, Academic Press, San Francisco (1980).

[5] D.R. MacGorman and W.D. Rust, The electrical nature of storms, Oxford Univ. Press, New York (1998).

[6] T. Marshall, W. Rust, and M. Stolzenburg, J. Geophys. Res., 100, 1001 (1995).
[7] V.V. Alexeenko et al, Phys.Lett. 301A, 299 (2002).

[8] Y.Muraki et al, Phys. Rev. D69, 123010 (2004).

[9] A.V. Gurevich et al, Phys. Lett. 325A, 389 (2004).

[10] A.V. Gurevich and K.P. Zybin, Phys. Usp., 44 (11), 1119 (2001).

[11] A.V. Gurevich and K.P. Zybin, Phys.Lett. 329, 341 (2004); A.V. Gurevich, Yu.V. Medvedev and K.P. Zybin, Phys.Lett. 329, 348 (2004);

[12] S.J. Sciutto, Proc. 27th ICRC (Hamburg) 1, 237 (2001). See also http://www.fisica.unlp.edu.ar/auger/aires/ 